\title{
A PULSAR SURVEY OF 18 SUPERNOVA REMNANTS
}

\author{
Peter W. Gorham, ${ }^{1}$ Paul S. Ray, ${ }^{2}$ Stuart B. Anderson, ${ }^{3}$ Shrinivas R. Kulkarni, ${ }^{4}$ \\ AND Thomas A. Prince 5 \\ Division of Physics, Mathematics, and Astronomy, California Institute of Technology, Pasadena, CA 91125 \\ Received 1995 April 27; accepted 1995 August 18
}

\begin{abstract}
We have performed a pulsar search of 18 known or probable supernova remnants with the Arecibo $305 \mathrm{~m}$ radio telescope at 430 and $1420 \mathrm{MHz}$. The remnants were selected to have angular sizes less than or of order a few telescope beam diameters $\left(\sim 10^{\prime}\right.$ at $430 \mathrm{MHz}$ and $\sim 3^{\prime}$ at $\left.1420 \mathrm{MHz}\right)$. In some cases, larger remnants with centrally brightened cores of this scale were also included. The observations were sensitive to pulsars with periods $\gtrsim 1 \mathrm{~ms}$ and flux densities as low as $\sim 0.2 \mathrm{mJy}$. The targets included two probable plerionic objects and several shell-type remnants with centrally brightened X-ray profiles. No new pulsars were discovered in the survey, and we discuss some of the selection effects that make the interpretation of pulsar surveys of supernova remnants problematic. Effects of high pulsar velocities, uncertain distances, and high remnant brightness temperatures make it difficult to exclude the presence of a pulsar in most remnants.
\end{abstract}

Subject headings: pulsars: general - supernova remnants

\section{INTRODUCTION}

In spite of the strong evidence that supernovae produce the neutron stars which become pulsars, the role of pulsars in the formation and evolution of supernova remnants (SNRs) and, conversely, the role of supernova remnants in producing (or obscuring) the observed properties of pulsars are poorly understood. Several known radio and X-ray pulsars are believed to be directly associated with SNRs. There is evidence that an additional 15-20 SNRs contain a "central engine," which is likely to be either a neutron star or black hole (Seward 1990). Although the list of SNR-pulsar associations is growing, the corresponding growth of the list of SNRs still outpaces it, and at present only $\sim 15 \%$ of the known Galactic SNRs show any evidence that a compact object was produced in the supernova event.

Table 1 lists 18 proposed pulsar-SNR associations. SNRs are classified (see Weiler \& Sramek 1988 for a review) as plerions (P), which show a filled-center, flat-spectrum nebula presumably powered by a compact object; shells (S), which show basically empty shells of emission; and composites (C), which show a shell in addition to a central plerion. The pulsar velocities are estimated by the distance to the remnant, the angular distance from the geometric center of the remnant, and either the pulsar characteristic age or the estimated remnant age. Because many of these quantities are difficult to estimate and prone to systematic errors, the quoted velocities should be taken as indicative rather than as precise measurements. The table also includes pulsar name, associated SNR, pulsar characteristic age $\left(\tau_{c}=P / 2 \dot{P}\right)$, approximate distance to the pulsar as derived from the dispersion measure (DM) and the

\footnotetext{
${ }^{1}$ Present address: Department of Physics and Astronomy, University of Hawaii, Honolulu, HI 96822; gorham@fermion.phys.hawaii.edu.

${ }^{2}$ Present address: Remote Sensing Division, Code 7213, Naval Research Laboratory, Washington, DC 20375; paulr@rira.nrl.navy.mil.

3 E-mail: sba@ccsf.caltech.edu.

4 E-mail: srk@astro.caltech.edu.

5 E-mail: prince@srl.caltech.edu.
}

Galactic electron density model of Taylor \& Cordes (1993), pulse period (p), pulsar flux(s) at 430 and $1400 \mathrm{MHz}, \mathrm{DM}$, and references to the literature.

There are three primary criteria on which associations of pulsars with SNRs are judged: positional alignment, distance, and age. Several of the associations do not have very good positional alignment, but this is usually explained by postulating very high birth velocities for pulsars. Some of the associations require extremely high velocities $\left(\gtrsim 1000 \mathrm{~km} \mathrm{~s}^{-1}\right)$ to be imparted to the pulsar by the supernova explosion, while most require only a few hundred $\mathrm{km} \mathrm{s}^{-1}$, which is typical of the older pulsar population (Lyne \& Lorimer 1994). For most of these pulsars, the velocities are rather uncertain because of the difficulty in measuring distances and ages of SNRs. However, the possibility of very high pulsar velocities introduces a clear selection effect into pulsar surveys of SNRs.

Observational evidence for pulsar-SNR associations comes from several different sources. First, the Crab and Vela were found in plerionic remnants which obviously required a central engine (Staelin \& Reifenstein 1968; Large, Vaughan, \& Mills 1968). Then, X-ray data from the Einstein Observatory resulted in several more pulsar discoveries in SNRs (Seward \& Harnden 1982; Seward, Harnden, \& Helfand 1984). Recently, progress has been made with additional radio observations of SNRs near young pulsars that resulted in new remnants being discovered or older remnants found to be larger than originally suspected, which made some associations more plausible (Caswell et al. 1992; McAdam, Osborne, \& Parkinson 1993; Routledge \& Vaneldik 1988). In addition, continued directed and undirected searches have resulted in the discovery of several other pulsars associated with SNRs (Wolszczan, Cordes, \& Dewey 1991; Anderson et al. 1994, for example).

We have performed a pulsar search of 18 known or probable SNRs with the Arecibo $305 \mathrm{~m}$ radio telescope at 430 and 1420 $\mathrm{MHz}$. To our knowledge, the only previous published search of comparable scope and sensitivity for radio pulsars associated with SNRs is that of Manchester, D'Amico, \& Tuohy (1985), which used the Parkes $64 \mathrm{~m}$ telescope, covering mainly sources 
TABLE 1

Known Pulsars with Proposed Supernova Remnant Associations

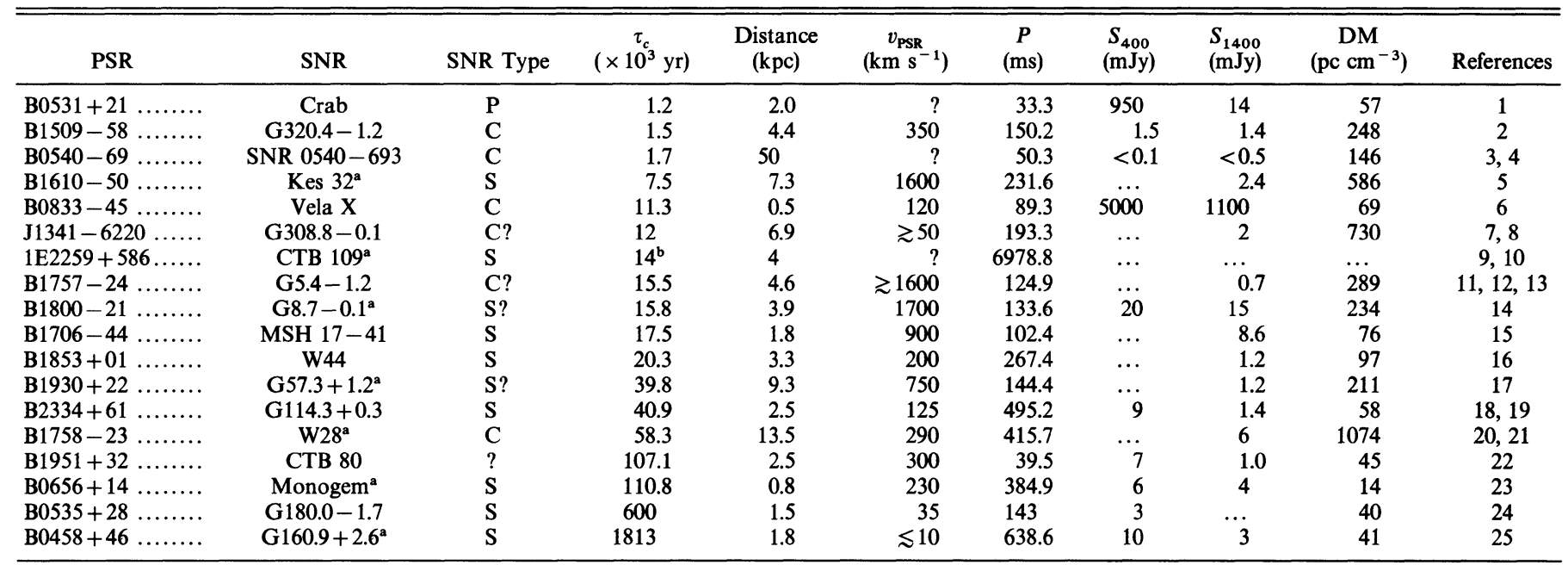

a These associations are uncertain.

${ }^{b}$ Remnant age, not pulsar characteristic age.

REFERENCES.- (1) Staelin \& Reifenstein 1968; (2) Seward \& Harnden 1982; (3) Seward, Harnden, \& Helfand 1984; (4) Manchester et al. 1994; (5) Caraveo 1993; (6) Large, Vaughan, \& Mills 1968; (7) Caswell et al. 1992; (8) Kaspi et al. 1992; (9) Gregory \& Fahlman 1980; (10) Fahlman \& Gregory 1981; (11) Manchester, D’Amico, \& Tuohy 1985; (12) Frail \& Kulkarni 1991; (13) Manchester et al. 1991; (14) Kassim \& Weiler 1990; (15) McAdam, Osborne, \& Parkinson 1993; (16) Wolszczan, Cordes, \& Dewey 1991;(17) Routledge \& Vaneldik 1988; (18) Kulkarni et al. 1993;(19) Fürst, Reich, \& Seiradakis 1993; (20) Frail, Kulkarni, \& Vasisht 1993; (21) Kaspi et al. 1993; (22) Kulkarni et al. 1988; (23) Cordova et al. 1989;(24) Anderson et al. 1994; (25) Leahy \& Roger 1991.

in the southern sky. A recent, unpublished search using the Arecibo telescope yielded a pulsar, PSR B1853+01, in the SNR W44, reported by Wolszczan et al. (1991).

\subsection{Rationale for Sensitive Pulsar Searches toward SNRs}

Until recently, it was believed that shell-type remnants could not contain young, fast pulsars (Narayan \& Schaudt 1988). However, the discovery of pulsars like PSR B1853+01 in W44 and PSR B2334+61 in G114.3+0.3 shows the importance of continued pulsar searches in shell-type remnants. Several of these recent associations with shell-type remnants have fluxes $\$ 2 \mathrm{mJy}$ at $1400 \mathrm{MHz}$. This strongly suggests that success in finding more pulsars in SNRs requires very sensitive searches, e.g., searches with large telescopes (such as Arecibo) and long integration times.

While most early associations were in plerionic or composite SNRs, the current list of associations has a majority of shelltype remnants (see Table 1). Narayan \& Schaudt (1988) argue that pulsars with reasonably fast rotation rates (such as those expected, and found, in SNRs) may have beaming factors close to unity. The detection of pulsars with short initial periods in MSH 17-41, G180.0-1.7, W44, and others implies that shelltype SNRs may contain pulsars likely to be beamed toward Earth. Thus, if adequate sensitivity can be achieved and if other selection effects (discussed below) can be minimized then, in principle, one should be able to detect pulsars in many SNRs.

\subsection{Searches and Selection Effects}

Several pulsar search selection effects in SNR surveys are competing, so that efforts to minimize one selection effect may exacerbate another. For example, SNRs of angular scale larger than the beam of a single-dish telescope must be observed by mosaic techniques; thus the observing time required to cover the area of the SNR increases as the square of the ratio of the remnant diameter to the beam diameter. An alternative is to observe at a lower frequency where the beam is larger; however, this reduces the sensitivity at higher dispersion measures due to both instrumental pulse broadening and scattering effects. On the other hand, steep spectrum pulsars may produce much more signal in a lower frequency observation, in spite of the increased system noise due to the SNR background. For a typical SNR, the spectral index is roughly -0.6 (see Green 1993), compared to a range of values of -0.5 to -2.0 for pulsars. However, the break-even spectral index depends both on the brightness temperature of the SNR and its shape, making it difficult to judge the optimal observation frequency a priori.

Recent work (Lyne \& Lorimer 1994) has demonstrated that pulsars are born with mean birth velocity of $450 \mathrm{~km} \mathrm{~s}^{-1}$, much higher than was previously believed. For remnants whose age is $\gtrsim 10^{5} \mathrm{yr}$, or younger for pulsars with very high birth velocities, the proper motion of a high-velocity pulsar may have moved it close to or beyond the expanding shell of the remnant. Cases have been made for associations that require pulsar velocities of up to $1600 \mathrm{~km} \mathrm{~s}^{-1}$ (Johnston et al. 1995; Caraveo 1993). These high-velocity pulsars move quickly out of their remnants; within a few million years they have moved out of range of most surveys and populate an extended Galactic halo.

A published pulsar survey of SNRs is that performed by Manchester et al. (1985) using the $64 \mathrm{~m}$ Parkes telescope at a frequency of $1400 \mathrm{MHz}$. This survey was sensitive to pulsars with periods greater than $10 \mathrm{~ms}$ and had a minimum detectable flux density of typically $\sim 1 \mathrm{mJy}$ for periods above $\sim 300 \mathrm{~ms}$ and $\mathrm{DM} \leq 1600 \mathrm{pc} \mathrm{cm}^{-3}$. Since the minimum detectable flux density (MDF) depends linearly on the sky temperature (see $\S 3.1$ ), a bright SNR will increase the MDF significantly, reducing the survey sensitivity. For typical SNR flux densities, this increase in the MDF may be a factor of 5 or more above the nominal cold-sky MDF. This explains the fact that the Parkes survey did not detect the W44 pulsar (1.2 mJy at 1400 
$\mathrm{MHz}$ ) in two different observations. Given the $\sim 200 \mathrm{Jy}$ flux density of the remnant at this frequency, the sky temperature observed was at least a factor of 2 greater than the receiver temperature. The pulsar, which is $\sim 7^{\prime}$ below the remnant centroid, was also near the half-power point of the telescope beam. This example indicates that determination of the completeness of any SNR survey is complicated because selection effects associated with beam size and the SNR background can introduce wide variations in the MDF.

Manchester et al. (1985) observed 53 SNRs and 59 other objects, many of which are accessible only from southern hemisphere sites. Four pulsars, one of which was already known as an X-ray pulsar, were discovered among the observed SNRs. For the remaining three pulsars, the association of each with the SNR was judged by Manchester et al. to be most likely a chance alignment, based on discrepancies between the positions of the pulsars relative to the remnants and the derived distances from the observed dispersion measures relative to those estimated independently for the remnants (usually from $\mathrm{H}$ I absorption). Based on further measurements, all three of these pulsars have turned out to be young, and all three now have proposed SNR associations, although one (PSR B1758-23) is quite controversial.

\section{SEARCH STRATEGY, OBSERVATIONS, AND ANALYSIS}

\subsection{Search Strategy}

The present search was performed on a selected set of SNRs observable from Arecibo (i.e., $-2^{\circ}<\delta<39^{\circ}$ ) and which could be covered fairly completely with a small number of beams, either at 430 or $1420 \mathrm{MHz}$. By using long observations (20 minutes to $2 \mathrm{hr}$ ) with rapid sampling $(0.5-1 \mathrm{~ms})$ we achieved high sensitivity ( $\$ 0.5 \mathrm{mJy}$ ) down to several millisecond periods. The sample of SNRs included at least two plerionic SNRs, a number of SNRs from the $2.7 \mathrm{GHz}$ Effelsberg Galactic plane survey (Reich et al. 1984), and several previously known SNRs with associated X-ray emission, filled center X-ray or radio morphologies, or associated compact radio sources. Table 2 provides a source list, with nominal centroid positions, spectral indices, extrapolated flux densities, approximate sizes, and distances from Green (1995) or Reich et al. (1984).

\subsection{Observations}

The observations were made 1988 December 24-28, 1989 July 6-15, 1989 September 11-20, and 1991 March 28-April 1 using the $305 \mathrm{~m}$ transit dish at Arecibo, Puerto Rico. The data were recorded in a manner identical to that described by Anderson et al. (1990), except that in some instances a sample integration time of $1 \mathrm{~ms}$, rather than $0.5 \mathrm{~ms}$, was used. The duration of the observations varied from $\sim 20$ minutes to $\sim 2$ hr per source transit, and several of the sources were observed more than once. The objects were usually observed at 430 $\mathrm{MHz}$ if the conditions allowed; however, severe radio interference problems often made $430 \mathrm{MHz}$ observations impractical, and in these cases $1420 \mathrm{MHz}$ observations were done instead. Certain interference bands appear in power spectra for almost all the data; for example, a moderately broadband noise signal at the first 3-4 harmonics of $0.32-0.35 \mathrm{~Hz}$ effectively eliminates any sensitivity at these frequencies. Also, most of the observations show power at $60 \mathrm{~Hz}$ and its harmonics.

Table 3 gives a log of the observations. The repeated entries for the same source in column (1) are either repeated observations or observations at a different beam position within the same remnant if the size of the remnant was larger than the telescope beam. The position of the beam center is given in columns (6) and (7). Column (11) gives an approximate value of the mean zenith angle of the source during the observation and is relevant to estimates of the telescope sensitivity.

The gain of the telescope at the zenith was $18 \mathrm{~K} \mathrm{Jy}^{-1}$ at 430 $\mathrm{MHz}$, and $8 \mathrm{~K} \mathrm{Jy}^{-1}$ at $1420 \mathrm{MHz}$ (NAIC 1989). The system temperatures were $60-70 \mathrm{~K}$ at $430 \mathrm{MHz}$ for the two polarization channels, and $40 \mathrm{~K}$ at $1420 \mathrm{MHz}$. At large zenith angles $\left(\sim 20^{\circ}\right)$ the gain at both frequencies decreases by $\sim 40 \%$, and the system temperatures increase by about a factor of 2 , due both to the decrease in effective aperture, and illumination of the warm ground.

\subsection{Data Analysis}

The pulse search used standard power spectral analysis. A time series is formed by converting the correlator output into 128 individual frequency channels and combining these into a single time series assuming a dispersive time delay across the band due to a trial value of the dispersion measure (see Ander-

TABLE 2

SUPERNOVA REMNANTS INCLUDED IN THE SEARCH

\begin{tabular}{|c|c|c|c|c|c|c|c|c|}
\hline Source & Name & R.A. (1950) & Decl. (1950) & Size & Type & Spectral Index & $1 \underset{(\mathrm{GH})}{ } F_{v}$ & $\begin{array}{l}\text { Distance } \\
\quad(\mathrm{kpc})\end{array}$ \\
\hline $30.7+1.0 \ldots \ldots$ & & $18^{\mathrm{h}} 42^{\mathrm{m}} 10^{\mathrm{s}}$ & $-01^{\circ} 35^{\prime}$ & $24^{\prime} \times 18^{\prime}$ & S? & -0.4 & 6 & \\
\hline $31.9+0.0 \ldots \ldots$ & 3C 391 & 184650 & -0059 & 5 & $\mathbf{S}$ & -0.55 & 24 & 8.5 \\
\hline $33.6+0.1 \ldots \ldots$ & Kes 79 & $\begin{array}{lll}18 & 50 & 15\end{array}$ & +0037 & 10 & S & -0.5 & 22 & 10 \\
\hline $36.6-0.7 \ldots \ldots$ & & 185805 & +0252 & $25 ?$ & S? & -0.5 & 0.7 & \\
\hline $39.2-0.3 \ldots \ldots$ & 3C 396 & $1901 \quad 40$ & +0523 & $8 \times 6$ & $\mathrm{~S}$ & -0.6 & 18 & $\gtrsim 7.7$ \\
\hline $39.5+0.6 \ldots \ldots$ & & $\begin{array}{lll}18 & 59 & 41\end{array}$ & +0602 & 12 & $\mathrm{C}$ & -0.15 & 3 & \\
\hline $41.1-0.3 \ldots \ldots$ & $3 \mathrm{C} 397$ & 190508 & +0703 & $4.5 \times 2.5$ & $\mathrm{~s}$ & -0.48 & 22 & $\gtrsim 7.5$ \\
\hline $41.4+0.4 \ldots \ldots$ & & $\begin{array}{lll}19 & 03 \quad 13\end{array}$ & +0740 & $12 ?$ & $?$ & -0.3 & 6 & \\
\hline $43.3-0.2 \ldots \ldots$ & W49B & $1908 \quad 44$ & +0901 & $4 \times 3$ & $\mathbf{S}$ & -0.5 & 38 & 10 \\
\hline $45.7-0.4 \ldots \ldots$ & & 191405 & +1104 & 22 & $\mathbf{S}$ & -0.3 & 4 & \\
\hline $49.2-0.7 \ldots \ldots$ & W51 & 192130 & +1400 & $25 ?$ & S? & $-0.3 ?$ & 160 & 4.5 \\
\hline $53.6-2.2 \ldots \ldots$ & $3 \mathrm{C} 400.2$ & 193630 & +1708 & 28 & $\mathrm{~S}$ & -0.6 & 8 & 6.7 \\
\hline $54.1+0.3 \ldots \ldots$ & $\ldots$ & $\begin{array}{lll}19 & 28 & 28\end{array}$ & +1846 & 1.5 & P? & -0.13 & 0.5 & \\
\hline $65.2+5.7 \ldots \ldots$ & $\ldots$ & 193100 & +3105 & $310 \times 240$ & S? & -0.6 & $52 ?$ & 0.8 \\
\hline $65.7+1.2 \ldots \ldots$ & DA 495 & $19 \quad 50 \quad 10$ & +2918 & 18 & $\mathrm{C} ?$ & -0.6 & 5 & $\ldots$ \\
\hline $73.9+0.9 \ldots \ldots$ & & $2012 \quad 20$ & +3603 & $22 ?$ & S? & -0.3 & $9 ?$ & $\ldots$ \\
\hline $74.0-8.5 \ldots \ldots$ & Cyg loop & 204900 & +3030 & $230 \times 160$ & $\mathbf{S}$ & Varies & 210 & 0.77 \\
\hline $74.9+1.2 \ldots \ldots$ & CTB 87 & $2014 \quad 10$ & +3703 & $8 \times 6$ & $\mathrm{P}$ & -0.24 & 9 & 12 \\
\hline
\end{tabular}


TABLE 3

SNR OBSERVATION LOG

\begin{tabular}{|c|c|c|c|c|c|c|c|c|c|c|}
\hline $\begin{array}{l}\text { Source } \\
\text { (1) }\end{array}$ & $\begin{array}{l}\text { Reference } \\
\text { Position } \\
\text { (2) }\end{array}$ & $\begin{array}{c}\text { Day } \\
\text { GMT } \\
(3)\end{array}$ & $\begin{array}{c}\text { Year } \\
(4)\end{array}$ & $\begin{array}{c}T \\
(\min ) \\
(5)\end{array}$ & $\begin{array}{c}\text { R.A. } 1950.0 \\
\text { (6) }\end{array}$ & $\begin{array}{c}\text { Decl. } 1950.0 \\
(7)\end{array}$ & $\begin{array}{c}v \\
(\mathrm{MHz}) \\
(8)\end{array}$ & $\begin{array}{c}B \\
(\mathrm{MHz}) \\
(9)\end{array}$ & $\begin{array}{c}t \\
(\mathrm{~ms}) \\
(10)\end{array}$ & $\begin{array}{c}\langle\mathrm{ZA}\rangle \\
(11)\end{array}$ \\
\hline $30.7+1.0$. & A & 189 & 1989 & 45 & $18^{\mathrm{h}} 41^{\mathrm{m}} 12^{\mathrm{s}} .9$ & $-01^{\circ} 32^{\prime} 38$ & 1418 & 40 & 0.5 & $20^{\circ}$ \\
\hline $30.7+1.0 \ldots \ldots$ & B & 193 & 1989 & 45 & $1840 \quad 46.2$ & -013420 & 1418 & 40 & 0.5 & 20 \\
\hline $30.7+1.0 \ldots \ldots$ & $\mathrm{C}$ & 90 & 1991 & 46 & $1841 \quad 17.5$ & -013720 & 1411 & 40 & 0.5 & 20 \\
\hline $31.9+0.0 \ldots \ldots$ & $\ldots$ & 91 & 1991 & 70 & 184653.0 & -005945 & 1411 & 40 & 0.5 & 20 \\
\hline $33.6+0.1 \ldots \ldots$ & $\ldots$ & 262 & 1989 & 22 & $1850 \quad 04.0$ & 003521 & 1408 & 40 & 1.0 & 19 \\
\hline $36.6-0.7 \ldots \ldots$ & $\ldots$ & 254 & 1989 & 45 & 185753.4 & 025841 & 430 & 10 & 0.5 & 16 \\
\hline $39.2-0.3 \ldots \ldots$ & $\ldots$ & 88 & 1991 & 70 & 190133.6 & 052235 & 1411 & 40 & 0.5 & 14 \\
\hline $39.5+0.6 \ldots \ldots$ & $\ldots$ & 195 & 1989 & 70 & 185908.7 & 060058 & 430 & 10 & 0.5 & 13 \\
\hline $41.1-0.3 \ldots \ldots$ & $\therefore$ & 88 & 1991 & 70 & $\begin{array}{lll}19 & 05 & 10.1\end{array}$ & 070400 & 1411 & 40 & 0.5 & 12 \\
\hline $41.1-0.3 \ldots \ldots$ & $\ldots$ & 194 & 1989 & 70 & 190510.0 & 070345 & 1418 & 40 & 0.5 & 13 \\
\hline $41.4+0.4 \ldots \ldots$ & A & 191 & 1989 & 75 & $\begin{array}{lll}19 & 03 & 13.0\end{array}$ & 073954 & 430 & 10 & 0.5 & 11 \\
\hline $41.4+0.4 \ldots \ldots$ & B & 192 & 1989 & 75 & $1903 \quad 34.0$ & 074329 & 430 & 10 & 0.5 & 11 \\
\hline $41.4+0.4 \ldots \ldots$ & $\mathrm{C}$ & 196 & 1989 & 70 & $1903 \quad 24.0$ & 074142 & 430 & 10 & 0.5 & 11 \\
\hline $43.3-0.2 \ldots \ldots$ & $\ldots$ & 187 & 1989 & 70 & $1908 \quad 44.3$ & 090110 & 430 & 10 & 0.5 & 11 \\
\hline $45.7-0.4 \ldots \ldots$ & A & 258 & 1989 & 35 & 191421.8 & 110210 & 430 & 10 & 1.0 & 14 \\
\hline $45.7-0.4 \ldots \ldots$ & B & 259 & 1989 & 24 & $\begin{array}{lll}19 & 13 & 32.3\end{array}$ & 110804 & 1408 & 40 & 1.0 & 11 \\
\hline $45.7-0.4 \ldots \ldots$ & $\mathrm{C}$ & 258 & 1989 & 28 & 191400.3 & 111436 & 1408 & 40 & 1.0 & 16 \\
\hline $49.2-0.7 \ldots \ldots$ & A & 87 & 1991 & 45 & 192049.0 & 140810 & 430 & 10 & 0.5 & 15 \\
\hline $49.2-0.7 \ldots \ldots$ & B & 87 & 1991 & 70 & $1921 \quad 11.2$ & 140000 & 430 & 10 & 0.5 & 6 \\
\hline $49.2-0.7 \ldots \ldots$ & B & 91 & 1991 & 70 & $1921 \quad 11.2$ & 140000 & 1411 & 40 & 0.5 & 8 \\
\hline $53.6-2.2 \ldots \ldots$ & $\ldots$ & 90 & 1991 & 45 & $\begin{array}{lll}19 & 35 & 48.8\end{array}$ & 171126 & 430 & 10 & 0.5 & 4 \\
\hline $54.1+0.3 \ldots \ldots$ & $\ldots$ & 361 & 1988 & 45 & $1928 \quad 17.5$ & 184542 & 430 & 10 & 0.5 & 3 \\
\hline $54.1+0.3 \ldots \ldots$ & $\ldots$ & 188 & 1989 & 148 & $1928 \quad 17.5$ & 184542 & 430 & 10 & 0.5 & 6 \\
\hline $54.1+0.3 \ldots \ldots$ & $\ldots$ & 260 & 1989 & 30 & $1928 \quad 17.0$ & 184550 & 1408 & 40 & 1.0 & 3 \\
\hline $65.3+5.7 \ldots \ldots$ & $\ldots$ & 262 & 1989 & 30 & $1933 \quad 36.0$ & 300200 & 1408 & 40 & 1.0 & 12 \\
\hline $65.7+1.2 \ldots \ldots$ & $\ldots$ & 193 & 1989 & 90 & $1950 \quad 10.7$ & 291815 & 430 & 10 & 0.5 & 12 \\
\hline $73.9+0.9 \ldots \ldots$ & $\mathbf{A}$ & 187 & 1989 & 45 & 201142.5 & 360525 & 430 & 10 & 0.5 & 18 \\
\hline $73.9+0.9 \ldots \ldots$ & B & 257 & 1989 & 20 & 201145.6 & 355941 & 430 & 10 & 0.5 & 18 \\
\hline $73.9+0.9 \ldots \ldots$ & BC & 191 & 1989 & 45 & $\begin{array}{lll}20 & 12 & 03.0\end{array}$ & 355846 & 430 & 10 & 0.5 & 18 \\
\hline $73.9+0.9 \ldots \ldots$ & BC & 192 & 1989 & 45 & $\begin{array}{lll}20 & 12 & 03.0\end{array}$ & 355846 & 430 & 10 & 0.5 & 18 \\
\hline $73.9+0.9 \ldots \ldots$ & $\mathrm{C}$ & 196 & 1989 & 45 & $2012 \quad 20.4$ & 355751 & 1418 & 40 & 0.5 & 18 \\
\hline $73.9+0.9 \ldots \ldots$ & D & 195 & 1989 & 70 & $2012 \quad 59.1$ & 355209 & 430 & 10 & 0.5 & 18 \\
\hline $73.9+0.9 \ldots \ldots$ & D & 258 & 1989 & 30 & $2012 \quad 59.1$ & 355210 & 430 & 10 & 0.5 & 18 \\
\hline $74.0-8.5 \ldots \ldots$ & $\ldots$ & 89 & 1991 & 50 & 204933.9 & 305137 & 430 & 10 & 0.5 & 19 \\
\hline $74.9+1.2 \ldots \ldots$ & $\ldots$ & 363 & 1988 & 45 & $2014 \quad 11.8$ & 370250 & 430 & 10 & 0.5 & 18 \\
\hline $74.9+1.2 \ldots \ldots$ & $\ldots$ & 190 & 1989 & 45 & $2014 \quad 14.0$ & 370400 & 430 & 10 & 0.5 & 18 \\
\hline $74.9+1.2 \ldots \ldots$ & $\ldots$ & 254 & 1989 & 45 & $2014 \quad 14.0$ & 370400 & 431 & 10 & 0.5 & 18 \\
\hline
\end{tabular}

son 1993). The time series used varied from $2^{20}-2^{24}$ samples in length, depending on the duration of the observation and the sampling interval used. The power spectra were computed and searched using the concurrent supercomputers of the Caltech Concurrent Supercomputing Facility (CCSF) and the Concurrent Supercomputing Consortium (CSCC). The initial data analysis was done on the CCSF nCUBE/10, and later analysis was done on the CSCC Intel Touchstone Delta and the CCSF nCUBE $/ 2$.

Standard spectral analysis techniques for pulsar searches, such as normalizing the power spectra using the local mean value from a boxcar average, and summing of harmonically related portions of the spectrum, were employed to maximize the sensitivity to short duty cycle pulses.

Dispersion measure values up to $1500 \mathrm{pc} \mathrm{cm}^{-3}$ were used for the $430 \mathrm{MHz}$ data. At this DM, the pulse broadening is equal to $\sim 12 \mathrm{~ms}$ per channel in the correlator, and the DM value corresponds to distances well outside the galaxy for the mean Galactic electron density (Taylor \& Cordes 1993). Higher values toward an individual source are possible if there is significant $\mathrm{H}$ II column density; however, interstellar scattering strongly reduces the sensitivity for $430 \mathrm{MHz}$ observations at DM values higher than this (Sutton 1971). At $1420 \mathrm{MHz}$, scattering effects are usually not important for DM values below a few times $10^{3} \mathrm{pc} \mathrm{cm}^{-3}$, although this varies with the line of sight.
The step size used for the DM trials for each observing frequency was chosen to retain uniform sensitivity to pulsars up to the Nyquist frequency of the sampling. In the $430 \mathrm{MHz}$ data sets the pulse broadening across one of the 128 frequency channels becomes larger than $2 \mathrm{~ms}$ for DM values above $\sim 200$ $\mathrm{pc} \mathrm{cm}^{-3}$. Therefore, the time series for large DM values were resampled to $2 \mathrm{~ms}$ sampling time. While significantly reducing the computational time, this resampling does not affect the sensitivity to pulsars with period longer than $\sim 8 \mathrm{~ms}$. Shorter period pulsar signals are strongly degraded by dispersion and scattering and could not have been detected anyway.

During the sequence of observations in this program, a search for globular cluster pulsars was also conducted using identical data reduction and analysis software. The globular cluster search was successful, yielding a total of four binary systems and seven isolated pulsars in the clusters M13, M15, M53, and NGC 6760 (Anderson et al. 1990; Anderson 1993; Kulkarni et al. 1991; Prince et al. 1991). These results provided a cross-calibration on the SNR search sensitivity, and allowed us to assess the effective detection threshold in the analysis.

\section{RESULTS}

\subsection{Minimum Detectable Flux Density}

The minimum detectable flux density (MDF) for a radio pulsar search depends directly on sky and receiver temperature, inversely on the square root of the duration of the 
observation, and directly on the square root of the observed pulse duty cycle. The pulse duty cycle is not known a priori. The observed pulse duty cycle may also be broadened by dispersion or other effects; thus the MDF also increases with increasing DM. A general expression for the MDF is given by Stokes et al. (1986) and in a slightly different form by Biggs \& Lyne (1992):

$$
S_{\min }=K\left[\frac{T_{\mathrm{rec}}+T_{\mathrm{sky}}(l, b)+T_{\mathrm{sp}}(\mathrm{ZA})}{G\left(N_{p} B t\right)^{1 / 2}}\right]\left(\frac{w_{\mathrm{eff}}}{P-w_{\mathrm{eff}}}\right)^{1 / 2},
$$

where the factor $K \sim 12$ is empirically determined from detection of known pulsars and expresses the analysis detection threshold $(\sim 8 \sigma)$. $T_{\text {rec }}, T_{\text {sky }}(l, b)$, and $T_{\text {sp }}(\mathrm{ZA})$ are the receiver temperature, sky temperature (depending on Galactic longitude and latitude), and effective spillover temperature (depending on the zenith angle), respectively. Here $G$ is the telescope gain in units of $\mathrm{K} \mathrm{Jy}^{-1}, N_{p}$ the number of polarization senses received, $B$ the total bandwidth in $\mathrm{MHz}$, and $t$ the total duration of the observation in seconds. The rightmost term in equation (1) gives the dependence on pulse duty cycle, with $P$ the pulse period, and $w_{\text {eff }}$ the effective pulse width:

$$
w_{\mathrm{eff}}=\left[w^{2}+\beta^{2} \tau^{2}+\left(\frac{\tau \mathrm{DM}}{\mathrm{DM}_{0}}\right)^{2}\right]^{1 / 2},
$$

where $w$ is the intrinsic pulse width, $\tau$ is the sampling interval in seconds, $\beta \simeq 2$ is related to the time constant of the postdetection smoothing filter, and $\mathrm{DM}_{0}$ is the dispersion measure value at which the pulse delay across the bandpass of a single narrow-frequency channel equals the sampling interval. This value is approximately

$$
\mathrm{DM}_{0}=1.2 \times 10^{-4} \frac{\tau v^{3}}{\Delta v} \mathrm{pc} \mathrm{cm}^{-3}
$$

where $v$ and $\Delta v$ are the observing frequency and channel bandwidth, respectively, both in $\mathrm{MHz}$. This formula is an approximation for a smooth profile of width $w$. If the shape differs very much from a Gaussian profile, then the harmonic content may be somewhat different, but dispersed pulsars typically have fairly smooth profiles.

Figure 1 plots $S_{\min }$ for a range of parameters encountered in this survey. Unless otherwise indicated, each of the plots use the following parameters: $\mathrm{DM}=300 \mathrm{pc} \mathrm{cm}-3$; pulse duty cycle (fraction of the pulse period when the pulse is "on") $\delta=0.10$; sampling interval $\tau=0.5 \mathrm{~ms}$; duration of observation $t=2800 \mathrm{~s}$; mean zenith angle $\langle\mathrm{ZA}\rangle=15^{\circ}$. For the effective sky temperature due to the SNR, a spectral index of -0.6 was assumed, with a $430 \mathrm{MHz}$ flux density of $15 \mathrm{Jy}$. This implies $T_{\text {sky }}=300 \mathrm{~K}(430 \mathrm{MHz})$ and $150 \mathrm{~K}(1400 \mathrm{MHz})$ where a beam filling factor of unity is assumed. This flux density is meant to represent the typical SNR included in the search. It should be noted that there is a great deal of variability in the sky temperature from remnant to remnant, and from pointing to pointing within a particular remnant. In each case, the solid curves correspond to the $430 \mathrm{MHz}$ results, and the dashed curves correspond to the $1400 \mathrm{MHz}$ results.

Figure $1 a$ shows the sensitivity variations for the three different sampling intervals used. Figure $1 b$ shows variation for different average zenith angles. The variation with $\mathrm{ZA}$ is caused by two factors: the spillover effects increase with increasing zenith angle, and the system gain decreases with increasing zenith angle as the effective aperture is truncated. Figure $1 c$ shows the variation for three different values of the DM, and Figure $1 d$ shows the change for differing values of the pulse duty cycle.

During observations of G39.5 +0.6, a signal was detected at a pulse period of $746 \mathrm{~ms}$, from the known pulsar PSR B1900+05, which was $\sim 19^{\prime}$ from the $430 \mathrm{MHz}$ beam center. The position corresponded to a sidelobe of the beam with a gain of $-12 \mathrm{~dB}$ relative to the beam center, at a zenith angle of $\sim 13^{\circ}$, thus giving $\sim 5 \%$ of the zenith gain at beam center. PSR B1900 + 05 has a $400 \mathrm{MHz}$ flux density of $20 \mathrm{mJy}$ in the sidelobe, giving an equivalent beam-center flux of $\sim 1 \mathrm{mJy}$. The pulsar was detected in its first 18 harmonics with an average of $\sim 8$ times the noise power in each harmonic, at a DM of $176 \mathrm{pc}$ $\mathrm{cm}^{-3}$. The pulse width for this pulsar is $26 \mathrm{~ms}$, giving a duty cycle of 0.03 . Taking into account the position of PSR $\mathrm{B} 1900+05$ relative to the beam center, and using equations (1) and (2), we obtain a sensitivity estimate of $0.7 \mathrm{mJy}$ at the beam center, providing some independent validation of the results in Figure 1.

\subsection{Flux Density Limits}

Table 4 gives limits for average pulsar flux density, using the minimum detectable flux densities estimated from equation (1) and the known properties of the remnants. No attempt was made to correct for the inhomogeneity of the sky temperature of the objects, although some corrections were made when the beam filling factors were obviously small so that the net background sky temperature was much lower than that of the remnant.

The observations at different frequencies and at different positions within the remnant are each given distinct limits in Table 4, and the fraction of the remnant solid angle that was covered by the observation is also indicated. The values for pulsar parameters used in determining the limits are $P=30$ $\mathrm{ms}, \mathrm{DM}=300 \mathrm{pc} \mathrm{cm}{ }^{-3}$, and duty cycle $\delta=0.1$. Limits for values other than the nominal values may be obtained from Figure 1 or by scaling with equation (1). Limits for distinct observations of the same beam position have not been combined in Table 4, but are quoted separately.

\section{DISCUSSION}

Flux limits for surveys of SNRs are rather hard to interpret in terms of making a definite statement that a pulsar was not born in the supernova. Distances to SNRs are difficult to constrain, and their ages are similarly uncertain. This significantly affects both luminosity and velocity estimates. Pulsar distances are believed to be somewhat better, but there is not a simple, empirical law for predicting pulsar radio luminosities. So, it is hard to place a luminosity limit for a particular remnant, and it is not clear what luminosity a pulsar of the remnant age would be expected to have. For remnants that are not very young, a high-velocity pulsar might be outside the remnant radio shell.

The median luminosity of the pulsars in Table 1 is $\sim 80 \mathrm{mJy}$ $\mathrm{kpc}^{2}$ using the DM-derived distances and estimates of the 400 $\mathrm{MHz}$ flux densities; they range from $\sim 5 \mathrm{mJy} \mathrm{kpc}^{2}$ for PSR $\mathrm{B} 0656+14$ and PSR B0535 + 28 to $3800 \mathrm{mJy} \mathrm{kpc}^{2}$ for the Crab pulsar. The obvious selection effect in this sample implies that the median luminosity for the whole population of pulsars in SNRs may considerably lower. For a typical flux limit of 0.3 mJy at $430 \mathrm{MHz}$ from Table 4 and a distance from 7 to $10 \mathrm{kpc}$, this survey places pulsar luminosity limits from 15 to $30 \mathrm{mJy}$ $\mathrm{kpc}^{2}$. The limit is much better for the few closer remnants; 

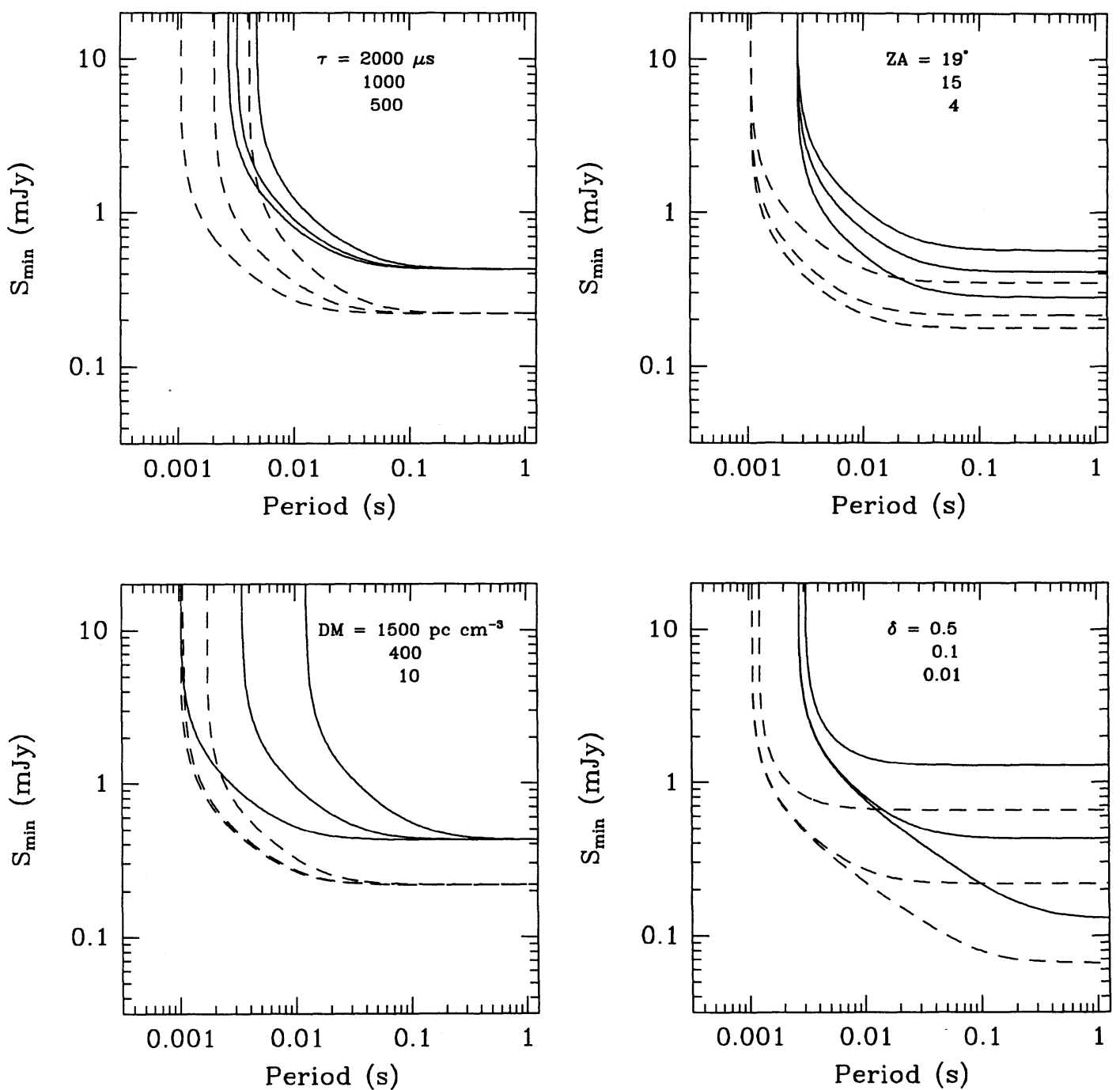

Fig. 1.-Minimum detectable flux density $S_{\min }$ as a function of pulsar period, for a range of parameters used in this survey, computed from eq. (1) in the text. Unless otherwise indicated by the notes in the upper right of each panel, the plots use the following parameters: DM $=300 \mathrm{pc} \mathrm{cm}^{-3} ;$ pulse duty cycle $\delta=0.10$; sampling interval $\tau=0.5 \mathrm{~ms}$; duration of observation $t=2800 \mathrm{~s}$; mean zenith angle $\langle\mathrm{ZA}\rangle=15^{\circ}$. For the effective sky temperature due to the SNR, a spectral index of -0.6 was assumed, with a $430 \mathrm{MHz}$ flux density of $15 \mathrm{Jy}$. This implies $T_{\text {sky }}=300 \mathrm{~K}(430 \mathrm{MHz})$ and $150 \mathrm{~K}(1400 \mathrm{MHz})$ where a beam filling factor of unity is assumed. (a) Variation of $S_{\min }$ with sampling interval. (b) Variation of $S_{\min }$ with mean source zenith angle during the observation. (c) Variation of $S_{\min }$ with dispersion measure. (d) Variation of $S_{\min }$ with pulse duty cycle. In each case, the solid curves correspond to the $430 \mathrm{MHz}$ results, and the dashed curve the $1400 \mathrm{MHz}$ results, and the order of the curves follows that of the indicated parameters in each panel.

eight of the remnants have uncertain distance estımates, preventing the calculation of luminosity limits.

The mean distance estimates for those SNR whose diameters fall within one telescope beam in this survey is $\sim 10 \mathrm{kpc}$ (Green 1995). At this distance the $267 \mathrm{~ms}$ pulsar in W44 would appear at a flux density of $0.15 \mathrm{mJy}$ at $1400 \mathrm{MHz}$, and W44 itself would have a $1 \mathrm{GHz}$ flux density of $\sim 25 \mathrm{mJy}$ and a diameter of $5^{\prime}$. These values for the size and diameter are very similar to those actually observed for a number of the SNRs surveyed here, such as G31.9+0.0, G41.1 - 0.3, and G43.3-0.2. At 1400 $\mathrm{MHz}$, the effective sky temperature due to these remnants is $\sim 150 \mathrm{~K}$. From equation (1), using the $55 \mathrm{~K}$ system temperature for the $1420 \mathrm{MHz}$ feed at Arecibo (zenith angle $=15^{\circ}$ ) and a 70 minute observation, the W44 pulsar would be detectable as long as the pulse duty cycle were less than 0.06 , corresponding to a pulse width of $16 \mathrm{~ms}$ at $267 \mathrm{~ms}$ pulse period. The majority of observed pulsars have intrinsic duty cycles within the range between $U . U \angle$ and $U .1 \mathrm{U}$, altnougn the ooserveu value will be increased by interstellar scattering at the high DMs expected for most of the remnants included in this search, and such variation will introduce a clear selection effect on detectability.

The effective sky temperature at $430 \mathrm{MHz}$ for these remnants is $\sim 300 \mathrm{~K}$. In this case, the effective sky temperature dominates the sensitivity in equation (1). For a 70 minute observation and a duty cycle of $0.1, S_{\min } \geq 0.5 \mathrm{mJy}$. Thus a spectral index of roughly -1.0 or steeper is required for a pulsar like the pulsar in W44 to be detectable at $430 \mathrm{MHz}$ when placed at a distance of $10 \mathrm{kpc}$. Such a spectral index is not unusual for pulsars as a whole; the spectral index of the W44 pulsar is -1.7 .

If we accept the results of Narayan \& Schaudt (1988), that beaming effects are not important for young pulsars in SNRs, then the limits obtained here for G74.9+1.2 (CTB 87), which is 
TABLE 4

\begin{tabular}{|c|c|c|c|c|c|}
\hline $\begin{array}{c}\text { Source } \\
\text { (1) }\end{array}$ & $\begin{array}{l}\text { Reference } \\
\text { Position } \\
\text { (2) }\end{array}$ & $\begin{array}{c}430 \mathrm{MHz} \\
\text { Limit } \\
(\mathrm{mJy}) \\
(3)\end{array}$ & $\begin{array}{l}\text { Percent of Area } \\
\text { Covered } \\
(430 \mathrm{MHz}) \\
\text { (4) }\end{array}$ & $\begin{array}{c}1400 \mathrm{MHz} \\
\text { Limit } \\
(\mathrm{mJy}) \\
(5)\end{array}$ & $\begin{array}{c}\text { Percent of Area } \\
\text { Covered } \\
(1400 \mathrm{MHz}) \\
(6)\end{array}$ \\
\hline $30.7+1.0 \ldots \ldots$ & A & $\ldots$ & $\ldots$ & 0.3 & 3 \\
\hline $30.7+1.0 \ldots \ldots$ & B & $\ldots$ & $\ldots$ & 0.3 & 3 \\
\hline $30.7+1.0 \ldots \ldots$ & $\mathrm{C}$ & $\ldots$ & $\ldots$ & 0.3 & 3 \\
\hline $31.9+0.0 \ldots \ldots$ & $\ldots$ & $\ldots$ & $\ldots$ & 0.8 & 45 \\
\hline $33.6+0.1 \ldots \ldots$ & A & $\ldots$ & $\ldots$ & 0.7 & 10 \\
\hline $36.6-0.7 \ldots \ldots$ & $\ldots$ & 0.2 & 15 & $\ldots$ & $\ldots$ \\
\hline $39.2-0.3 \ldots \ldots$ & $\ldots$ & $\ldots$ & $\ldots$ & 0.3 & 23 \\
\hline $39.5+0.6 \ldots \ldots$ & $\ldots$ & 0.2 & 70 & $\ldots$ & $\ldots$ \\
\hline $41.1-0.3 \ldots \ldots$ & $\ldots$ & $\ldots$ & $\ldots$ & 0.35 & 80 \\
\hline $41.1-0.3 \ldots \ldots$ & $\ldots$ & $\ldots$ & $\ldots$ & 0.35 & 80 \\
\hline $41.4+0.4 \ldots \ldots$ & A & 0.3 & 70 & $\ldots$ & $\ldots$ \\
\hline $41.4+0.4 \ldots \ldots$ & B & 0.3 & 70 & $\ldots$ & $\ldots$ \\
\hline $41.4+0.4 \ldots \ldots$ & $\mathrm{C}$ & 0.3 & 70 & $\ldots$ & $\ldots$ \\
\hline $43.3-0.2 \ldots \ldots$ & $\ldots$ & 1.2 & 100 & $\ldots$ & $\ldots$ \\
\hline $45.7-0.4 \ldots \ldots$ & A & 0.4 & 20 & $\ldots$ & $\cdots$ \\
\hline $45.7-0.4 \ldots \ldots$ & B & $\ldots$ & $\ldots$ & 0.3 & 3 \\
\hline $45.7-0.4 \ldots \ldots$ & $\mathrm{C}$ & $\ldots$ & $\ldots$ & 0.3 & 3 \\
\hline $49.2-0.7 \ldots \ldots$ & A & 1.0 & 15 & $\ldots$ & $\ldots$ \\
\hline $49.2-0.7 \ldots \ldots$ & B & 0.6 & 15 & 0.5 & 2 \\
\hline $53.6-2.2 \ldots \ldots$ & $\ldots$ & 0.2 & 13 & $\ldots$ & $\ldots$ \\
\hline $54.1+0.3 \ldots \ldots$ & $\ldots$ & 0.5 & 100 & $\ldots$ & $\ldots$ \\
\hline $54.1+0.3 \ldots \ldots$ & $\ldots$ & 0.3 & 100 & 0.3 & 100 \\
\hline $65.3+5.7 \ldots \ldots$ & $\ldots$ & $\ldots$ & $\ldots$ & 0.1 & $<1$ \\
\hline $65.7+1.2 \ldots \ldots$ & $\ldots$ & 0.3 & 30 & $\ldots$ & $\ldots$ \\
\hline $73.9+0: 9 \ldots \ldots$ & A & 0.3 & 20 & $\ldots$ & $\ldots$ \\
\hline $73.9+0.9 \ldots \ldots$ & B & 0.5 & 20 & $\ldots$ & $\ldots$ \\
\hline $73.9+0.9 \ldots \ldots$ & BC & 0.3 & 20 & $\cdots$ & $\cdots$ \\
\hline $73.9+0.9 \ldots \ldots$ & $\mathrm{BC}$ & 0.3 & 20 & $\ldots$ & $\ldots$ \\
\hline $73.9+0.9 \ldots \ldots$ & $\mathrm{C}$ & $\ldots$ & $\ldots$ & 0.3 & 3 \\
\hline $73.9+0.9 \ldots \ldots$ & D & 0.2 & 20 & $\ldots$ & $\ldots$ \\
\hline $73.9+0.9 \ldots \ldots$ & $\mathrm{D}$ & 0.4 & 20 & $\cdots$ & $\cdots$ \\
\hline $74.0-8.5 \ldots \ldots$ & $\ldots$ & 0.3 & $<1$ & $\ldots$ & $\ldots$ \\
\hline $74.9+1.2 \ldots \ldots$ & $\ldots$ & 0.5 & 100 & $\ldots$ & $\ldots$ \\
\hline $74.9+1.2 \ldots \ldots$ & $\ldots$ & 0.4 & 100 & $\ldots$ & $\ldots$ \\
\hline $74.9+1.2 \ldots \ldots$ & $\ldots$ & 0.4 & 100 & $\ldots$ & $\ldots$ \\
\hline
\end{tabular}

classified as a plerion, give some indication of the aging of the pulsed intensity of such a pulsar. Using a distance of $12 \mathrm{kpc}$ for CTB 87, and $2 \mathrm{kpc}$ for the Crab Nebula, a Crab-like pulsar in CTB 87 would have $\sim 40$ times less flux density, or $\leq 20 \mathrm{mJy}$ at $430 \mathrm{MHz}$. The age of CTB 87 is probably at least 10 times that of the Crab, or $\sim 10 \times 10^{3} \mathrm{yr}$. Our limit $(\leq 0.5 \mathrm{mJy}$ for $P \geq 100$ $\mathrm{ms}$ and $\mathrm{DM} \leq 400 \mathrm{pc} \mathrm{cm}^{-3}$ ) implies that any pulsar in CTB 87 must be a factor of at least 40 less luminous in pulsations than the Crab pulsar at $430 \mathrm{MHz}$. Of course, without knowing the initial spin period and magnetic field of the presumed pulsar powering CTB 87 it is not possible to make a direct comparison with the Crab Pulsar.

One other remnant in our survey has been suggested to be plerionic in nature: G54.1 +0.3 (Velusamy \& Becker 1988). This object has a centrally brightened radio core with a high degree of linear polarization and is an X-ray source. Imaging the central portion of this SNR has been limited in resolution due to its compact size and faintness. The distance to G54.1 + 0.3 has been estimated with large uncertainty to be 17 $\mathrm{kpc}$. At this distance the Crab pulsar would have a flux density of $\sim 10 \mathrm{mJy}$ at $430 \mathrm{MHz}$, and the DM would be $\sim 400 \mathrm{pc}$ $\mathrm{cm}^{-3}$ (Taylor \& Cordes 1993), giving a pulse smearing of $\sim 5$ ms. The Crab pulsar would thus be about a factor of 20 above the minimum detectable flux density for this SNR, and the possibility of a relatively luminous pulsar in this remnant is not excluded by our limit if the distance is actually this large. Thus, further searches on this object should attempt to minimize the dispersion effects if possible.

Another remnant included in our search is SNR G33.6+0.1, which shows a compact radio peak with a coincident linear polarization maximum, and a suggestion of a flattening of the spectral index in the central region of the remnant. Our observations of G33.6+0.1, made without the benefit of the highresolution maps of Velusamy, Becker, \& Seward (1991), did not point directly at the radio maximum, but $1^{\prime} .0-1{ }^{\prime} .4$ to the southeast. The radio maximum is still within the $3 \mathrm{~dB}$ beam width of 3.3 of the $1408 \mathrm{MHz}$. Arecibo feed, but there is a $\sim 30 \%-40 \%$ reduction in gain relative to the beam center. This effect, combined with the increased apparent sky temperature on this SNR and the large zenith angle necessary to observe it from Arecibo, increase the limiting detectable flux density for this object to $\sim 0.3 \mathrm{mJy}$ for a $10 \%$ duty cycle pulse. This is probably insufficient to detect a W44-type pulsar in G33.6+0.1 unless the SNR is at the lower limit of its estimated range of distance $(\sim 7 \mathrm{kpc})$.

\section{CONCLUSIONS}

We conclude that the most prominent selection effects in detecting pulsars in SNRs are the severe sensitivity limits imposed by the large distances and intrinsic brightness tem- 
peratures of the majority of known objects or the Galactic plane in which they lie, and high birth velocities of radio pulsars, which cause many pulsars associated with SNRs to be off-center or even outside the remnant. It is evident that any theoretical estimates regarding the presence of pulsars in SNRs must consider carefully the loss of sensitivity that occurs in pulsar searches of SNRs compared to the more numerous sky surveys.

Future searches will require significant improvements in sensitivity in order to constrain the pulsar population in more distant remnants, as well as searching a larger solid angle to account for high pulsar velocities. A reduced system temperature from the newly installed ground screen at Arecibo, as well as improvements in correlator bandwidth and spectral sampling, along with requisite computing power to reduce the increased volume of data, will be necessary since constraints on observing time will probably continue to limit the maximum attainable duration of time series in similar survey projects. It appears that observations of Galactic plane SNRs should concentrate on higher frequencies than $430 \mathrm{MHz}$, despite the steep spectral index of many pulsars, since the effects of the high temperature of the Galactic plane and possible pulse smearing from scattering probably offset the improvement in sampling given by the larger beam diameter.

This work would not have been possible without the use of the Caltech nCUBE/10 operated by the Caltech Concurrent Supercomputing Facility. This research was performed in part using the Intel Touchstone Delta System operated by Caltech on behalf of the Concurrent Supercomputing Consortium. Access to this facility was provided by Caltech.

We thank the staff of Arecibo Observatory for help in performing the observations. The Arecibo Observatory is part of the National Astronomy and Ionosphere Center, which is operated by Cornell University under cooperative agreement with the National Science Foundation. This research was supported in part by NSF grant AST 90-20787. Basic research in precision pulsar astrophysics at the Naval Research Laboratory is supported by the Office of Naval Research. A portion of this work was performed while one of the authors (P. S. R.) held a National Research Council-NRL Research Associateship.

\section{REFERENCES}

Anderson, S. B. 1993, Ph.D. thesis, California Inst of Technology

Anderson, S. B., Cadwell, B. J., Wolszczan, A., \& Foster, R. S. 1994, IAU Circ., No. 6012

Anderson, S. B., Gorham, P. W., Kulkarni, S. R., Prince, T. A., \& Wolszczan, A. 1990 , Nature, 346,42

Biggs, J. D., \& Lyne, A. G. 1992, MNRAS, 254, 257

Caraveo, P. A. 1993, ApJ, 415, L111

Caswell, J. L., Kesteven, M. J., Stewart, R. T., Milne, D. K., \& Hanes, R. F. 1992, ApJ, 399, L151

Cordova, F. A., Hjellming, R. M., Mason, K. O., \& Middleditch, J. M. 1989, ApJ, 345, 451

Fahlman, G. G., \& Gregory, P. C. 1981, Nature, 293, 202

Frail, D. A., \& Kulkarni, S. R. 1991, Nature, 352, 785

Frail, D. A., Kulkarni, S. R., \& Vasisht, G. 1993, Nature, 365, 136

Fürst, E., Reich, W., \& Seiradakis, J. H. 1993, A\&A, 276, 470

Green, D. A. 1995, in IAU Colloq. 145, Supernovae and Supernova Remnants: ed. R. McCray \& Z. Wang (Cambridge: Cambridge Univ. Press), in press

Gregory, P. C., \& Fahlman, G. G. 1980, Nature, 287, 805

Johnston, S., Manchester, R. N., Lyne, A. G., Kaspi, V. M., \& D'Amico, N. 1995, A\&A, 795, 802

Kaspi, V. M., Lyne, A. G., Manchester, R. N., Johnston, S., D'Amico, N., \& Shemar, S. L. 1993, ApJ, 409, L57

Kaspi, V. M., Manchester, R. N., Johnston, S., Lyne, A. G., \& D’Amico, N. 1992, ApJ, 399, L155

Kassim, N. E., \& Weiler, K. W. 1990, Nature, 343, 146

Kulkarni, S. R., Anderson, S. B., Prince, T. A., \& Wolszczan, A. 1991, Nature, 349,47

Kulkarni, S. R., Clifton, T. C., Backer, D. C., Foster, R. S., Fruchter, A. S., \& Taylor, J. H. 1988, Nature, 331, 50

Kulkarni, S. R., Predehl, P., Hasinger, G., \& Aschenbach, B. 1993, Nature, 362 135
Large, M. I., Vaughan, A. E., \& Mills, B. Y. 1968, Nature, 220, 340

Leahy, D. A., \& Roger, R. S. 1991, AJ, 101, 1033

Lyne, A. G., \& Lorimer, D. R. 1994, Nature, 369, 127

Manchester, R. N., D'Amico, N., \& Tuohy, I. R. 1985, MNRAS, 212, 975

Manchester, R. N., Kaspi, V. M., Johnston, S., Lyne, A. G., \& D’Amico, N. 1991, MNRAS, 253, 7P

Manchester, R. N., Mar, D. P., Lyne, A. G., Kaspi, V. M., \& Johnston, S. 1994, ApJ, 403, L29

McAdam, W. B., Osborne, J. L., \& Parkinson, M. L. 1993, Nature, 361, 516

National Astronomy and Ionosphere Ctr., Cornell Univ. 1989, Arecibo Observatory User's Manual

Narayan, R., \& Schaudt, K. J. 1988, ApJ, 325, L43

Prince, T. A., Anderson, S. B., Kulkarni, S. R., \& Wolszczan, A. 1991, ApJ, 374, L41

Reich, W., Furst, E., Steffen, P., Reif, K., \& Haslam, C. G. T. 1984, A\&AS, 58, 197

Routledge, R. V., \& Vaneldik, J. F. 1988, ApJ, 326, 751

Seward, F. D. 1990, ApJS, 73, 781

Seward, F. D., \& Harnden, F. R. 1982, ApJ, 256, L45

Seward, F. D., Harnden, F. R., \& Helfand, D. J. 1984, ApJ, 287, L19

Staelin, D. H.,\& Reifenstein, E. C., III. 1968, Science, 162, 1481

Stokes, G. H., Segelstein, D. J., Taylor, J. H., \& Dewey, R. J. 1986, ApJ, 311, 694

Sutton, J. M. 1971, MNRAS, 155, 51

Taylor, J. H., \& Cordes, J. M. 1993, ApJ, 41 1, 674

Velusamy, T., \& Becker, R. H. 1988, AJ, 95, 1162

Velusamy, T., Becker, R. H., \& Seward, F. D. 1991, AJ, 102, 676

Weiler, K. W., \& Sramek, R. A. 1988, ARA\&A, 26, 295

Wolszczan, A., Cordes, J. M., \& Dewey, R. J. 1991, ApJ, 372, L99 